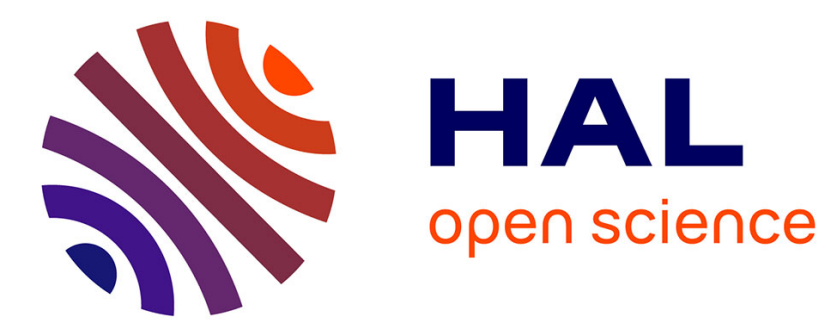

\title{
Un retour d'expérience graphique sur la crise cyclonique de 2017 aux Antilles
}

Elie Chevillot-Miot, Ingrid Canovas, Cheila Duarte-Colardelle, Christian Iasio, Thierry Winter, Valérie November

\section{- To cite this version:}

Elie Chevillot-Miot, Ingrid Canovas, Cheila Duarte-Colardelle, Christian Iasio, Thierry Winter, et al.. Un retour d'expérience graphique sur la crise cyclonique de 2017 aux Antilles. Annales des mines Série Responsabilité et environnement, 2020, 98, pp.41-47. hal-02959279

\section{HAL Id: hal-02959279 \\ https://hal.science/hal-02959279}

Submitted on 22 Oct 2020

HAL is a multi-disciplinary open access archive for the deposit and dissemination of scientific research documents, whether they are published or not. The documents may come from teaching and research institutions in France or abroad, or from public or private research centers.
L'archive ouverte pluridisciplinaire HAL, est destinée au dépôt et à la diffusion de documents scientifiques de niveau recherche, publiés ou non, émanant des établissements d'enseignement et de recherche français ou étrangers, des laboratoires publics ou privés. 


\section{RESPONSABILITÉ}

\&

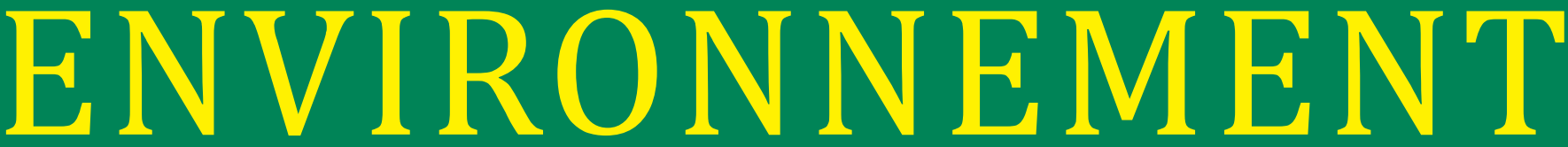

"Se défier du ton d'assurance qu'il est si facile de prendre et si dangereux d'écouter » Charles Coquebert, Journal des mines $n^{\circ} 1$, Vendémiaire An III (septembre 1794)

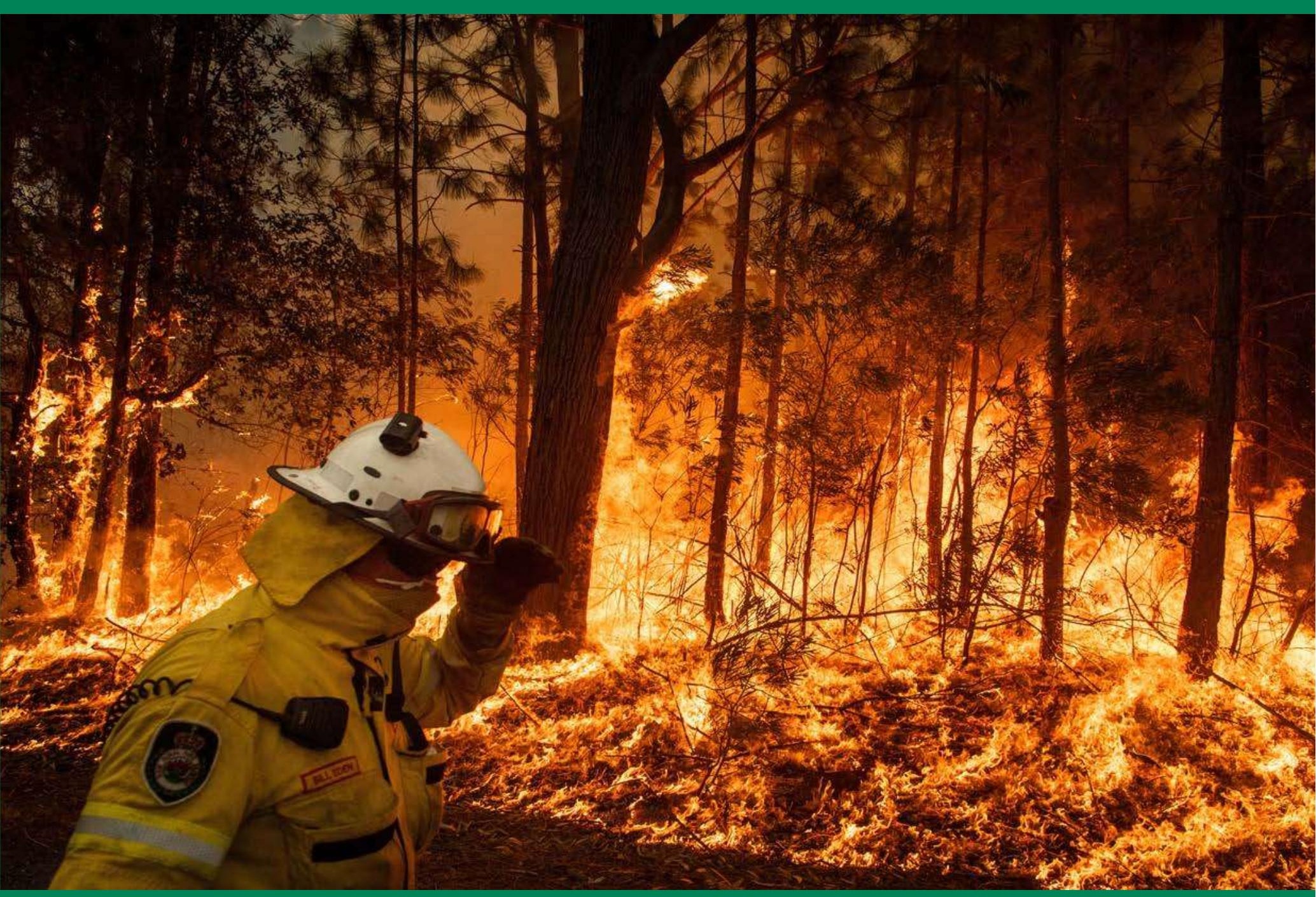

\section{Actualité de la catastrophe}




\section{ANNALES \\ DES MINES}

FONDÉES EN 1794

\section{Responsabilité \& Environnement}

ISSN 1268-4783

Série trimestrielle - n`98 - avril 2020

\section{Rédaction}

Conseil général de l'Économie (CGEIET),

Ministère de l'Économie et des Finances

120, rue de Bercy - Télédoc 797 - 75572 Paris Cedex 12

Tél : 0153185268

http://www.annales.org

François Valérian

Rédacteur en chef

Gérard Comby

Secrétaire général

Delphine Mantienne

Secrétaire générale adjointe

Liliane Crapanzano

Correctrice

Myriam Michaux

Webmestre / Maquettiste

\section{Membres du Comité de Rédaction}

Pierre Couveinhes

Président du Comité de rédaction

Ingénieur général des Mines honoraire

\section{Paul-Henri Bourrelier}

Ingénieur général des Mines honoraire,

Association française pour la prévention des catastrophes naturelles

\section{Mireille Campana}

Ingénieur général des Mines,

Conseil général de l’Économie

\section{Dominique Dron}

Ingénieur général des Mines,

Conseil général de l'Économie

\section{Pascal Dupuis}

Chef du service du climat et de l'efficacité énergétique, Direction générale de l'énergie et du climat, MTES

\section{Jérôme Goellner}

Chef du service des risques technologiques,

Direction générale de la prévention des risques, MTES

Jean-Luc Laurent

Ingénieur général des Mines honoraire

\section{Richard Lavergne \\ Conseil général de l’Économie}

Ministère de l'Économie et des Finances

Didier Pillet

Ingénieur général des Mines

Philippe Saint Raymond

Ingénieur général des Mines honoraire

Bruno Sauvalle

Ingénieur en chef des Mines, MINES ParisTech

Jacques Serris

Ingénieur général des Mines,

Conseil général de l’Économie

Claire Tutenuit

Déléguée générale d'Entreprises pour l'Environnement (EPE)

François Valérian

Rédacteur en chef des Annales des Mines

\section{Photo de couverture}

Combat contre l'incendie du Meroo National Park, Australie, 5 janvier 2020

(c) Matthew Abbott/The New York Times-REDUX-REA

Iconographie

Christine de Coninck

Abonnements et ventes

COM \& COM

Bâtiment Copernic - 20, avenue Édouard Herriot 92350 LE PLESSIS-ROBINSON

Alain Bruel

Tél. : 0140942222 - Fax : 0140942232

a.bruel@cometcom.fr

Mise en page : Nadine Namer

Impression : Printcorp

Éditeur Délégué :

FFE - 15, rue des Sablons - 75116 PARIS - www.ffe.fr

Fabrication : Aïda Pereira - 0153362046

aïda.pereira@belvederecom.fr

Régie publicitaire : Belvédère Com

Directeur de la publicité : Bruno Slama - 0140096617 bruno.slama@belvederecom.fr

La mention au regard de certaines illustrations du sigle " D. R. » correspond à des documents ou photographies pour lesquels nos recherches d'ayants droit ou d'héritiers se sont avérées infructueuses. 


\section{Actualité de la catastrophe}

04

Avant-propos

Paul-Henri BOURRELIER

06

Introduction

Maud DEVÈS

\section{La catastrophe : un concept protéiforme}

09

Apprendre du passé pour mieux faire face aux catastrophes

Emmanuel GARNIER

\section{4}

Tectonique et religions

Thierry GAUDIN

\section{8}

Les paradoxes de la prophétie de malheur

Critique de la collapsologie

Jean-Pierre DUPUY

\section{2}

Prospective, catastrophe et collapsologie Jacques THEYS

\section{Actualités de la catastrophe}

\section{9}

Les incendies de forêt catastrophiques

Éric RIGOLOT, Jean-Luc DUPUY,

François PIMONT et Julien RUFFAULT

\section{6}

Les défis soulevés par la reconstruction post-Irma Jérémy DESARTHE, Nicolas BAUDUCEAU et Antoine QUANTIN

\section{1}

Un retour d'expérience graphique sur la crise cyclonique de 2017 aux Antilles

Élie CHEVILLOT-MIOT, Ingrid CANOVAS, Cheila DUARTE-COLARDELLE, Christian IASIO, Thierry WINTER et Valérie NOVEMBER

\section{8}

La recherche française sur les risques et catastrophes naturels : bilan d'une décennie de financements de l'Agence nationale de la recherche (ANR) et perspectives d'avenir Anne LIEUTAUD, Maud DEVĖS, Nicolas ECKERT, Gilles GRANDJEAN, Mélanie PATEAU et Céline BILLIĖRE

\section{3}

WannaCry, une frayeur à l'échelle planétaire Jean-Luc AMINOT

60

Fukushima : décider en situation extrême Franck GUARNIERI et Sébastien TRAVADEL

\section{Agir ensemble face à la catastrophe}

\section{3}

La doctrine en matière de sûreté nucléaire :

une amélioration continue intégrant mieux la gestion d'un accident nucléaire

Jean-Luc LACHAUME et Sylvie CADET-MERCIER

\section{8}

Catastrophe et pilotage :

la sortie de tous nos "domaines de vol » Patrick LAGADEC

\section{2}

La résilience : opportunité ou fausse piste? Magali REGHEZZA

\section{7}

Catastrophes et sécurité sanitaire : aspects conceptuels et politiques Pr. William DAB

\section{1}

Recherche, expertise et décision face à la catastrophe : vers de nouveaux défis Maud DEVĖS

86

Catastrophes : qu'ont à dire les humanitaires ? François GRÜNEWALD 
Hors dossier

\section{2}

Les leviers d'action pour un mix énergétique propre et sûr au service de la transition énergétique dans les territoires

Florence CARRÉ, Karine ADAM,

Raymond COINTE, Olivier GENTILHOMME, Mathieu BRUGIDOU, Olivier GUILLAUME, Pauline GABILLET, Corinne GENDRON, Magali JAKUBOWICZ, Jean-Yves LEBER, Stéphane MOCANU, Isabelle MORETTI, Éric VIDALENC et Jacques VILLENEUVE

\section{6}

Le changement climatique :

quels enjeux pour les entreprises?

Godefroy GALAS et Angel PRIETO

\section{9}

Bilan énergétique de la France en 2018

Données définitives

Ministère de la Transition écologique et solidaire,

Commissariat général au Développement durable, Sous-direction des Statistiques de l'énergie (SDES)
103

Traductions des résumés

107

Biographies

Le dossier a été coordonné par Maud DEVĖS et Paul-Henri BOURRELIER 


\title{
Un retour d'expérience graphique sur la crise cyclonique de 2017 aux Antilles
}

\author{
Par Élie CHEVILLOT-MIOT \\ INHESJ, département Risques et Crises \\ Ingrid CANOVAS \\ LATTS, École des Ponts, Université Gustave Eiffel \\ Cheila DUARTE-COLARDELLE \\ INHESJ, département Risques et Crises \\ Christian IASIO et Thierry WINTER \\ BRGM \\ et Valérie NOVEMBER \\ LATTS, UMR 8134 CNRS, École des Ponts, Université Gustave Eiffel
}

\begin{abstract}
Réaliser le retour d'expérience d'une crise extrême, telle la crise cyclonique de 2017 aux Antilles françaises, est une procédure délicate et complexe. Elle nécessite non seulement d'intégrer les contraintes et incertitudes liées au passage de trois cyclones majeurs, mais également les effets des actions et décisions prises par l'important réseau des acteurs mobilisés à tous les niveaux d'intervention. La capitalisation d'expériences individuelles et collectives au sein d'un RETEX unique est donc primordiale pour optimiser la prise de décision en conduite de crise " hors-norme ", notamment dans un contexte de changement climatique aux effets imprédictibles. Dans cet article, nous proposons un retour d'expérience sous la forme d'une base de données graphique qui intègre des informations hétérogènes et descriptives des aléas, des actions menées, des interactions entre les services, etc. II s'agit, in fine, d'enrichir les connaissances et d'appuyer les décisions, à tous les niveaux (opérationnel, tactique et stratégique), tant dans le public que dans le privé.
\end{abstract}

\section{Introduction}

En conduite de crise, la compréhension des accrochages entre des contraintes de natures (incertitudes, multiples acteurs, biais cognitifs, etc.) et de dimensions variées (constantes spatio-temporelles, impact de l'événement et de la décision, etc.) est un enjeu primordial pour optimiser la prise de décision et l'anticipation en situation d'incertitude. En cela, les retours d'expérience revêtent une importance capitale bien que leur doctrine demeure modeste. Ils fournissent des réponses efficaces face à des événements futurs par une planification de mesures et d'actions de protection et de réduction des effets d'un aléa. Mais ils restent basés sur des phénomènes connus ou des crises « réglées" (maîtrisées, résolues). Peu d'entre eux sont axés sur les interactions entre les composantes « perception de la situation - Common Operational Picture - processus de décision " (Donahue et Tuohy, 2006 ; Wybo et
Laitiers, 2006) à tous les échelons du dispositif ORSEC (1) et des dispositifs privés.

Le retour d'expérience proposé dans le cadre du projet APRIL (2) s'attache 1) à étendre la portée de l'outil qu'il est pour en faire un support d'aide à la décision en situation extrême et à tous les niveaux (opérationnel, tactique et stratégique), afin d'en anticiper les effets pour sortir plus rapidement de la crise et préparer et engager au plus vite la phase de reconstruction. Pour cela, il s'appuie sur les témoignages des acteurs mobilisés lors des ouragans survenus en 2017 aux Antilles françaises, 2) lesquels sont intervenus dans un contexte exceptionnel regorgeant d'incertitudes liées aux phénomènes physiques, aux aléas

(1) Organisation de la réponse de sécurité civile.

(2) Optimiser l'anticipation et la prise de décision en situation de crises extrêmes pour maintenir la résilience de la société. 
secondaires (effets cascade) et aux fonctions techniques, organisationnelles et humaines sollicitées (Amalberti et Barricault, 1999 ; Bourdeaux et Gilbert, 1999). Les informations ont été ensuite 3 ) capitalisées dans une base de données de référence, à partir de laquelle une analyse de la performance des actions menées et des mesures prises (chemins décisionnels) a été finalement réalisée 4) à l'aide de graphes dynamiques.

\section{Le RETEX, une procédure primordiale pour se préparer aux risques et aux crises futures}

La procédure classique de retour d'expérience est institutionnalisée dans la loi de modernisation de la sécurité civile de 2004. Bien que devenue une priorité et une méthodologie harmonisée (MIAT, 2006), la formalisation de cette procédure (appelée REX, RETEX ou PEX) reste néanmoins modeste à tous les niveaux de décision tant publics que privés.

Des objectifs modestes, mais ambitieux...

Au sens opérationnel, le retour d'expérience est un outil qui permet d'analyser, a priori, des signaux faibles et, a posteriori, des événements perturbateurs réels ou fictifs, en considérant leurs caractéristiques physiques et leur déroulement spatio-temporel. II permet d'identifier le niveau de perturbation et les réponses apportées aux composantes techniques, humaines et organisationnelles, ainsi que le potentiel d'apprentissage positif comme négatif (Wybo et al., 2003 ; Gaillard, 2009 ; Morin, 2012 ; Casse, 2015). En conséquence, le retour d'expérience est supposé :

- améliorer la prédiction du phénomène et réduire ou éviter sa réalisation (Wildavsky, 1991) ;

- dégager des mesures de planification et des réponses, telles que des stratégies d'action, d'affectation des ressources, de reconstruction, de résilience d'un territoire ou d'une entreprise (Kruke et Olsen, 2005 ; Xiang et Zhaung, 2014) ;

- renforcer la coordination entre les gestionnaires de crise publics et/ou privés (Colardelle et Guinet, 2004) ;

- anticiper les prises de décisions en conduite de crise, pour un événement connu (Gautier, 2005 ; November et al., 2019).

Au sens stratégique, le retour d'expérience se doit de capter la dynamique des situations. II expose les efforts d'amélioration et évalue les effets des mesures prises individuellement et collectivement, lors de la gestion de l'événement perturbateur (Wybo, 2001 ; 2004).

Pour autant, ces deux sens sont interdépendants. Dès lors, le retour d'expérience doit s'attacher à analyser avec attention les décisions et actions perçues, leurs effets et leurs appréhensions, mais aussi les boucles de progrès (bonnes pratiques), lors de la conduite de crise. L'ensemble de ces éléments conditionnent l'efficience du retour d'expérience et participent à l'apprentissage et à la capitalisation des expériences individuelles et collectives d'une crise « réglée » (Amalberti et Barricault, 1999 ; Bourdeaux et Gilbert, 1999 ; Quiblier, 2007 ; Lalouette, 2008 ;
Morin, 2012). De fait, la préparation et les réponses apportées en situation de conduite de crise en sont optimisées (Kourosh et al., 2008 ; Cheema et al., 2016).

\section{... en dépit d'une mise en œuvre scabreuse}

La temporalité de la mise en œuvre d'une telle procédure constitue un facteur susceptible d'en affecter l'issue, et donc de mettre à mal ses ambitions. En effet, elle peut se réaliser " à chaud ", c'est-à-dire pendant la crise ou immédiatement après la sortie de crise (Baird et al., 1999 ; Vashdi et al., 2007 ; Godé, 2012). Cette démarche peut alors être perçue comme péjorative (exposition des erreurs), contraignante (perte de temps), ou comme une évaluation (enquête judiciaire) des capacités individuelles à faire face à la crise (Gilbert, 2001 ; MIAT, 2006). C'est pourquoi le retour d'expérience « à froid » est plus productif, dans la mesure où les acteurs impliqués souffrent moins du stress enduré et sont plus enclins à partager leur vécu, en raison d'un changement de poste, d'orientation politique, etc. Néanmoins, les récits peuvent ne pas refléter la réalité, en raison d'une perte de mémoire, la crainte du jugement, d'une perception angélique du déroulement des faits, etc. (Casse, 2015).

L'efficience du retour d'expérience dépend également du niveau de fiabilité des données à obtenir et de la diversité des sources d'information (acteurs). Si cette diversité tend à optimiser la remontée des informations, elle peut, à l'inverse, contribuer à leur fragmentation (Dechy et al., 2009). De plus, la confidentialité des retours d'expérience de crises majeures entrave leur formalisation et le partage transversal (Gaillard, 2005). Ils prennent le plus souvent la forme de rapports, tels les REX du portail ORSEC, peu exploités et reposant sur le déroulement factuel du phénomène, des moyens et actions engagés. Certains auteurs les ont cependant développé sous d'autres formes (numérique, cartographies dynamiques), pour en faire un véritable outil d'aide à la décision lors de crises futures (Morin, 2012 ; Pourinet et Creach, 2019) ou les rendre intelligibles pour un plus large public, par le biais de colloques, comme ce fut le cas pour les cyclones de $2017^{(3)}$.

Ces types de retours d'expérience sont adaptés à des crises "réglées ", connues, mais sont peu pertinents pour des crises dites " hors cadre ". Ils tiennent compte des éléments de contexte, des interactions et de leur interdépendance, ou encore de l'incertitude quant à la véracité des informations, lorsque certains repères habituels sont brouillés : défaillance ou rupture des circuits réglementés, multiplication des chaînes de décision (Guihou et al., 2006), du turn-over des agents expérimentés conduisant à une perte de transfert des expériences, etc. Au vu de ces éléments, une nouvelle conception du retour d'expérience semble se justifier. Le retour d'expérience classique nécessite d'être complété afin de rendre performant le principe du recours à un tel outil pour la conduite de crise " hors cadre".

(3) Le colloque «RETEXIRMA » de Résilience France ou encore le colloque " cyclones " des Géorisques de l'Université de Montpellier, organisés plus d'un an après les faits et ayant chacun invité des interlocuteurs divers (chercheurs, gestionnaires de crise publics et privés). 


\begin{tabular}{|c|c|c|c|}
\hline SANTÉ - Social & SÉcurité - SECours & LOGISTIQUE - URBANISME & Autres Services \\
\hline$\square \square$ Pôle Solidarité Famille & $\square \square$ Centre Secours Pompiers & $\square \square$ Port & $\square \square$ Archives \\
\hline$\square \square$ Agence Régionale Santé & $\square$ Gendarmerie & $\square \square$ Direction Mer & $\square \square$ Gestion Risques \\
\hline$\square \square$ Croix Rouge & $\square \square$ Police aux Frontières & $\square \square$ DEAL $^{(1)}$ & $\square \square$ Déchèterie \\
\hline$\square \square$ Service Médecine Urgence & $\square$ Douanes & $\square \square$ Police Urbanisme & $\square \square$ BRGM $^{(2)}$ \\
\hline EdUCATION - JUSTICE & OpÉrATEURs RÉsEAuX & PRÉfEcture & $\mathrm{CIC}-\mathrm{Coz}-\mathrm{Cog}_{\mathrm{C}}{ }^{(4)}$ \\
\hline$\square \square$ Rectorat & $\square \square$ EDF & $\square \square$ Communication & \\
\hline$\square \square$ Tribunal & $\begin{array}{l}\square \square \text { Orange } \\
\square \square \text { Dauphin Telecom }\end{array}$ & $\begin{array}{l}\square \square \text { Direction Cabinet } \\
\square \square \text { SIDPC }^{(3)}\end{array}$ & $\begin{array}{l}\text { Guadeloupe } \\
\text { Saint Martin }\end{array}$ \\
\hline
\end{tabular}

(i) Direction l'Environnement, de l'Amenagement et du Logement

(2) Bureau de Recherches Géologiques et Minières

Canovas I., Chevillot-Miot E. 2020

(3) Service Interministériel de Défense et de Protection Civile

(4) Cellule Interministérielle de Crise - Centre Opérationnel de Zone - Centre Opérationnel de Gestion Interministérielle des Crises

Figure 1 : Échantillon des services soumis à l'enquête.

\section{La nécessité d'un RETEX de la crise cyclonique 2017 aux Antilles françaises}

Notre étude s'est inscrite dans ce contexte de crise extrême, au cours de laquelle les décisions s'appuient non seulement sur une planification, mais aussi sur des réflexes, de l'adaptation et de l'anticipation. À partir de plusieurs retours d'expérience individuels, un RETEX global a été réalisé. Chacun d'eux a été analysé et traduit sous la forme d'une base de données relationnelle et graphique conçue pour alimenter un outil d'aide à l'anticipation et à la prise de décision en conduite de crise.

Rencontrer le réseau des acteurs mobilisés pour gérer la crise

Dans la nuit du mercredi 6 septembre, l'ouragan Irma (catégorie 5) traverse les îles de Saint-Martin et de Saint-Barthélemy, occasionnant des impacts sans précédent dans cette zone (onze morts et $95 \%$ des habitations impactées, voire pour certaines détruites). Sans impact direct, le passage de José, annoncé deux jours plus tard, a, quant à lui, entraîné un arrêt des interventions d'urgence post-Irma, le report des actions de préparation, de sécurisation des bâtiments et de mise à l'abri des personnes, et le blocage des transports maritimes et aériens. Moins de deux semaines après le passage d'Irma, l'œil du cyclone Maria passait à quelques kilomètres au sud de l'archipel de la Guadeloupe. S'il n'a apporté que des fortes précipitations sur Saint-Martin, le passage de Maria (catégorie 5) a en revanche occasionné en Guadeloupe des dégâts considérables sur le bâti des zones littorales et mis à l'épreuve l'organisation de la réponse, encore concentrée sur Saint-Martin.

Le passage successif de ces trois cyclones intenses a nécessité la mise en place d'une organisation complexe et exceptionnelle, impliquant la mobilisation de nombreux acteurs à différents niveaux d'intervention. Des RETEX ont été réalisés auprès d'un échantillon représentatif de ces services publics et privés - recueil de l'ordre de quarante témoignages (voir les Figures 1 ci-dessus et 2 de la page suivante) - de façon à retracer les chaînes de décision horizontales (intra-échelon) et verticales (inter-échelon), malgré un turn-over du personnel ${ }^{(4)}$, et ce dix-huit mois après les événements afin de :

- ne pas empiéter sur la préparation de la saison cyclonique de 2019 ;

- éviter les témoignages « à chaud », mais tout en s'assurant de la fiabilité de la mémoire des événements ;

- bénéficier d'une prise de recul et d'une analyse individuelle a posteriori ;

- étendre le suivi de la crise à celui des premiers temps de la reconstruction..

L'objectif multiple de ce retour d'expérience vise 1) à capitaliser l'expérience et le vécu des gestionnaires de la crise et de la reconstruction, et 2) à procéder à une analyse relationnelle, afin 3 ) d'identifier les actions et missions de chacun, les interactions entre les différents acteurs, la nature et le circuit des informations, les difficultés rencontrées, etc. Outre 4) des recommandations de bonne pratique, ce travail cherche 5) à identifier ce qui peut être anticipé, à chaque niveau de la chaîne de décision, et à tout instant du déroulement de la crise.

\section{Tirer des leçons du passé}

Les expériences individuelles, partagées et analysées par les gestionnaires de la crise, constituent le corpus de données à partir duquel une analyse relationnelle a été conduite, et une série de "bonnes pratiques " en a été extraite. Ces deux dernières étapes n'ont pas encore été initiées. Toutefois, certains moments forts et difficultés ont pu être révélés à l'issue d'entrevues effectuées sur l'île de Saint-Martin (voir la Figure 3 de la page suivante).

Cet inventaire initial permet de spécifier, voire de compléter la liste des faits déjà connus du grand public (presse, publications, etc.). II sera enrichi par la mise en relation de tous les récits et d'une analyse détaillée des effets de chaîne de chaque information, action ou décision identifiée, durant les phases d'alerte, de crise et de reconstruction.

(4) Six changements de poste en Cellule interministérielle de crise ont été identifiés et six changements de poste en outre-mer, conduisant, par voie de conséquence, à une perte importante de transfert de l'expérience. 


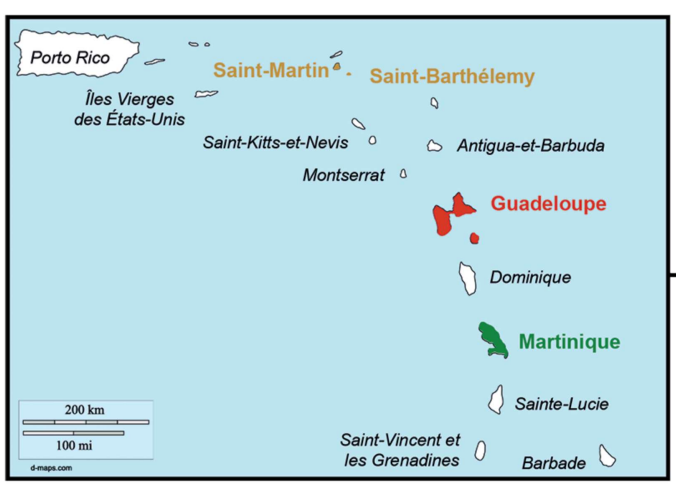

Réalisée par Chevillot-Miot É., 2020.
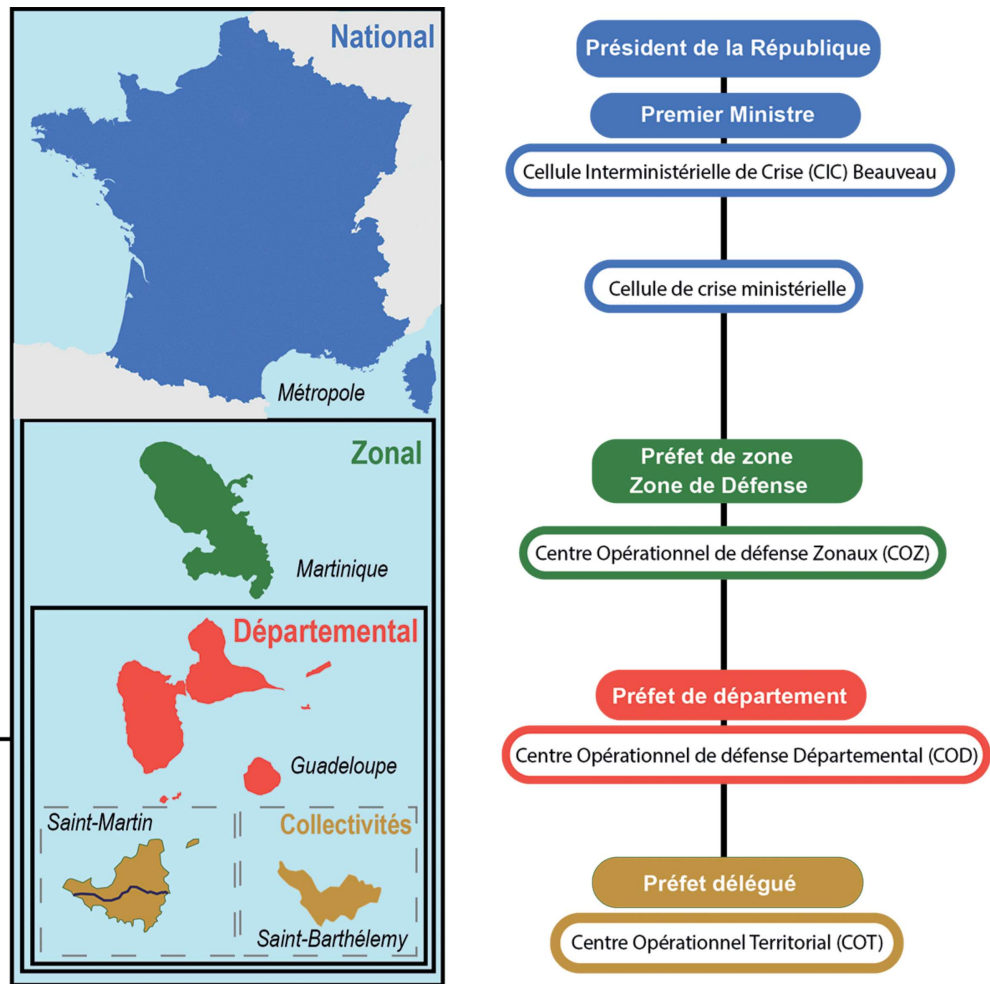

Figure 2 : Contexte géo-spatial du dispositif ORSEC mis en place lors des ouragans de 2017.

\section{Contrainte / Difficulté | Conséquence(s)}

Perte totale de communication

Nombreux pillages

Arrivées des renforts militaires

Visite Présidentielle et Ministérielle

Encombrement des routes

Gestion métropolitaine post-IRMA

Gestion des évacuations

Exposition de la famille des gestionnaires

Mutation du personnel en saison cyclonique

Méconnaissance ou sous-estimation du risque

Offre en abris sûrs de qualité/quantité insuffisante

Impact psychologique et soutien insuffisant

Refus des dons et des bénévoles
Isolement de l'île (population et services locaux de gestion de crise)

Expansion de rumeurs impossibles à démentir

Climat de crainte

Population effrayée et traumatisée

Climat de crainte

Soulagement

Sentiment de sécurité

Soulagement «nous n'avons pas été oubliés»

Dissipation momentanée des tensions

Interventions compliquées des services de sécurité et des secours Isolement de nombreuses personnes et de certains quartiers

Montée du racisme, par refus de l'expertise locale Crainte d'une "crise néocolonialiste»

Frustration liée à la priorisation des touristes et métropolitains Tensions sociales

Difficulté à concentrrer ses efforts sur la gestion de crise

Manque de préparation des gestionnaires du service public

Manque de préparation et de réaction de la population ayant connu LUIS et/ou GONZALO Indifférence des touristes aux alertes et consignes

Refus des personnes vulnérables de rejoindre les abris sûrs

Départ des métropolitains

Augmentation de la délinquance

Apparition de la violence intra-familiale - envers les femmes

Mise à mal de l'image de Saint-Martin : ingratitude vis-à-vis de la solidarité nationale

Canovas I, Chevillot-Miot E. 2020

Figure 3 : Les grands éléments relevant du RETEX IRMA, d'après les témoignages recueillis sur l'île de Saint-martin. 


\section{Améliorer le retour d'expérience des prises de décisions et optimiser la sortie de crise et la reconstruction}

Utilisation d'une base de données relationnelle orientée graphe pour organiser les données du RETEX

Chercher à apprendre non seulement des phénomènes les plus impactants, mais aussi des accidents mineurs et des quasi-accidents (ceux évités de justesse), est une opération complexe mais cruciale pour anticiper des événements majeurs à venir. La principale difficulté réside dans le traitement d'une masse d'informations très hétérogènes (quantitatives, qualitatives, géographiques, de perception, etc.), et plus particulièrement dans l'étape de rapprochement des différents fragments de données, afin d'obtenir une information capable de révéler le danger latent. À l'issue de la collecte, l'implémentation et l'harmonisation des données, une nouvelle couche d'informations originale peut être intégrée dans le système.

L'approche développée s'appuie sur la théorie des graphes. Étant une représentation mathématique des relations, le graphe est en effet adapté aux interprétations conceptuelles des systèmes complexes et au paramétrage de l'efficacité des pratiques de gestion des catastrophes. Les informations collectées y sont modélisées dans des relations par paires, entre " objets " : les sommets (nœuds ou points) sont reliés par des arêtes (liens ou lignes). La définition d'une ontologie qualifiant ces deux objets est capitale pour parvenir à la décomposition des informations initiales en composantes élémentaires, et pour per- mettre le raisonnement ou l'inférence. L'ontologie a été développée en utilisant les relations hiérarchiques et sémantiques restituées dans les entretiens.

\section{Construction du retour d'expérience de la crise cyclonique de 2017}

Le retour d'expérience de la crise cyclonique de 2017 se fonde sur la création d'un méta-modèle de base de données orientée graphe. L'exercice consiste à structurer l'ensemble des informations extraites des entretiens et d'autres sources de données au sein d'une base de données unique. Pour cela, le processus de déconvolution s'attache au préalable à décomposer les informations en éléments simples (nœuds et liens). Ces éléments, qui possèdent leurs propres attributs (dont la temporalité), sont ensuite regroupés dans des catégories ou ontologies, telles que :

- pour les nœuds : les institutions, les ressources, les messages, les perceptions, etc. ;

- et pour les relations : les actions, les décisions, les émotions, etc.

À l'issue de cette procédure, chaque chaîne d'action ou de décision peut être représentée sous sa forme élémentaire au sein d'un graphique (voir la Figure 4 ci-après) et chaque nouvelle information (issue d'un autre témoignage) peut y être implémentée en rattachant l'un de ses éléments à un nœud (ontologie) commun, s'il existe. En appliquant cette même méthode de décomposition aux informations contenues dans les entretiens, une représentation graphique (mapping) de l'ensemble de la réponse institutionnelle à la crise peut être obtenue.
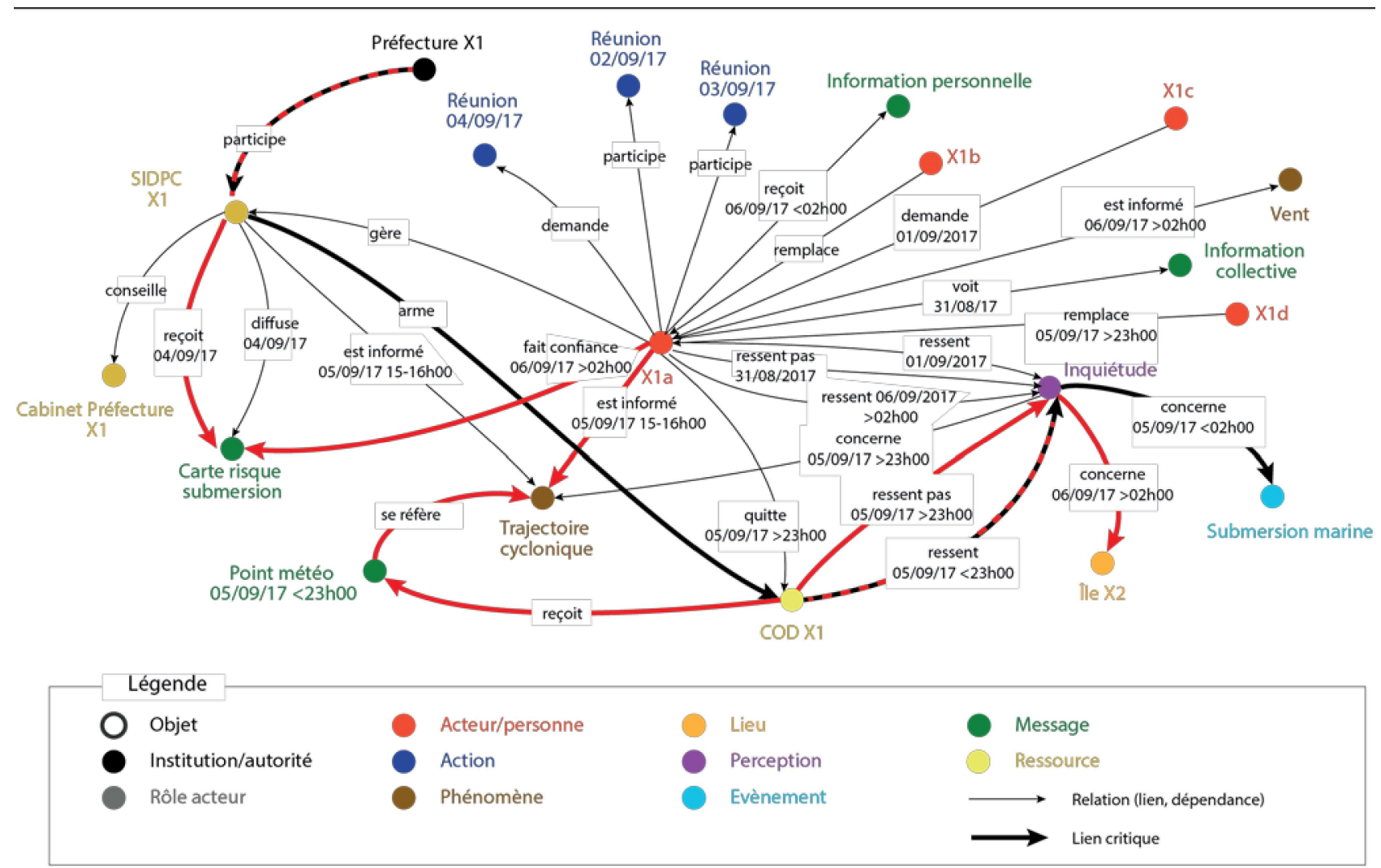

Figure 4 : Exemple de graphe restituant les relations «parenté à la trajectoire cyclonique », d'après le témoignage d'un interviewé. (source : Canovas I., Chevillot-Miot É. et lasio C. (2020)). 
Les résultats attendus de la méthode

L'organisation des informations et le type d'affichage fournis par la base de données orientée graphe mettent en lumière des relations de cause à effet qui n'auraient pu être décelées à partir d'une information ou d'une source d'information unique. C'est le cas par exemple du changement de niveau d'alerte en Guadeloupe : informée du passage en alerte rouge de la Martinique, la population de la Guadeloupe a exercé une pression telle qu'elle a conduit les autorités à faire également passer ce département en alerte rouge, avant l'échéance initialement fixée par la Préfecture, ce qui a été à l'origine de difficultés supplémentaires imprévues pour la population et la gestion de la crise elle-même.

Cette méthode présente surtout l'avantage de révéler des relations indirectes, comme des boucles d'information atypiques ou non protocolaires mais redondantes. Cela concernerait, par exemple, les circuits d'information informels ou non balisés, comme la demande d'une donnée spécifique formulée auprès d'un contact extérieur au réseau ou au canal réglementaire ou l'envoi spontané par ce même contact d'une telle donnée. Une fois reconnus, ces circuits pourraient conduire à une mise à jour des procédures afin d'améliorer, d'accélérer formellement le transfert des informations et de stimuler ainsi la prise de décision.

\section{Perspectives et conclusion}

Anticiper les effets d'un ouragan est une opération complexe et délicate. En raison de ses caractéristiques propres et des conditions extérieures, la dynamique de chaque phénomène s'accompagne d'un niveau d'incertitude qui persiste jusqu'à quelques heures avant son premier impact. Elle comprend cependant des phases (passage de l'œil) au cours desquelles il est possible d'intervenir pour en atténuer les effets. L'anticipation de ces dynamiques et des effets des actions ou décisions constitue dès lors un outil crucial pour la gestion de ces crises.

Ce travail d'anticipation repose sur l'alimentation de la phase pré-événement (anticipation) par la phase post-événement (prise de recul), en mettant en œuvre un continuum entre analyse et apprentissage. Ce retour d'expérience constitue un matériel singulier pour développer des outils destinés à améliorer la prise de décision et, à terme, la capacité d'anticipation. La capitalisation d'expériences individuelles, particulièrement celles des gestionnaires de crise publics et privés, par le biais d'entretiens, devrait permettre in fine de créer un prototype de cet outil d'aide à la décision et de développer une méthodologie de l'anticipation en conduite de crise, dans un contexte d'incertitude et de changements climatiques pouvant conduire à de nouvelles crises extrêmes, encore inimaginables à ce jour.

La structuration des informations au sein d'une base de données robuste, pertinente et évolutive est le résultat d'une collaboration entre des chercheurs issus des sciences naturelles, de l'ingénierie et des sciences humaines et sociales, afin de couvrir l'ensemble des disci- plines dans lesquelles s'inscrit la gestion de crise. Ainsi, la richesse des expertises réunies (théoriques, scientifiques, opérationnelles, etc.) devrait conduire à l'ajustement de certaines procédures et à des propositions de formation adaptées aux besoins réels des gestionnaires actuels et futurs dans des contextes changeants ou inconnus.

\section{Bibliographie}

AMALTERTI R. \& BARRICAULT C. (1999), "Fondements et limites du retour d'expérience ", Revue Annales des Ponts et Chaussées, $\mathrm{n}^{\circ} 91,67 \mathrm{p}$.

AZEMARD P., BATISTA D., BEAUDELOT L., MICHEL J.-L. \& MORTIER F. (2017), " Impacts de l'ouragan Irma sur les infrastructures à Saint-Martin ", rapport de synthèse du CEREMA.

BAIRD L., HOLLAND P. \& DEACON S. (1999), "Learning from action: Embedding more learning into the performance fast enough to make a difference", Organizational Dynamics, vol. $27, \mathrm{n}^{\circ} 4$, pp. 19-32.

BOURDEAUX I. \& GILBERT C. (1999), "Procédures de REX, d'apprentissage et de vigilances organisationnelles : approches croisées ", programme Risques collectifs et situations de crise, Grenoble, CNRS, septembre.

CASSE C. (2015), Concevoir un dispositif de retour d'expérience intégrant l'activité réflexive collective : un enjeu de sécurité dans les tunnels routiers, thèse de psychologie du travail et d'ergonomie, Université Grenoble Alpes, 395 p.

CHEEMA A. R., MEHMOOD A. \& IMRAN M. (2016), "Learning from the past: Analysis of disaster management structures, policies and institutions in Pakistan", Disaster Prevention and Management 25 (4), pp. 449-463.

COLARDELLE C. \& GUINET V. (2004), « Analyse de l'évolution de l'articulation organisationnelle - Préfet/Maire/Citoyen face à la crise », rapport pour le ministère de l'Intérieur, DDSC.

DECHY N., DIEN Y., LLORY M. \& MERAD M. (2009), "Les échecs organisationnels du retour d'expérience ", rapport d'étude du programme INERIS EAT DRA 71 - Évaluation des risques des systèmes industriels, $72 \mathrm{p}$.

DE LA TORRE Y. (2017), " Impacts du cyclone IRMA sur le littoral des "Îles du Nord" à Saint-Martin et Saint-Barthélemy ", rapport BRGM/RP-67291-FR, 20 III., 2 annexes, 29 p.

DUVAT V. (2008), " Le système du risque à Saint-Martin (Petites Antilles françaises) ", Développement durable et territoires, Catastrophes et Territoires, (11), $21 \mathrm{p}$.

DONAHUE A. \& TUOCHY R. (2006), "Lessons we don't learn: a study of lessons of disaster, why we repeat them, and how we can learn them", Homeland Security Affairs, vol. 2, n², 28 p., https://www.hsaj.org/articles/167

GAILLARD I. (2005), "État des connaissances bibliographiques sur les facteurs socioculturels de réussite ou d'échec du REX industriel ", Institut pour une Culture de la sécurité industrielle, 5 juillet, p. 33.

GAILLARD I. (2009), "S'organiser pour apprendre des perturbations : le retour d'expérience ", in DE TERSSAC G., BOISSIĖRES I. \& GAILLARD I. (coords), La Sécurité en action, Octarès, Toulouse, coll. "Le travail en débat », pp. 153-174.

GAUTIER A. (2005), "Retour d'expérience organisationnel et conceptualisation de l'action dans une perspective d'apprentissage pour les organisations ", Information Sciences for Decision Making 40, $704 \mathrm{p}$.

GILBERT C. (2001), " Retours d'expérience : le poids des contraintes ", Annales des Mines, Responsabilité \& Environne- 
ment, 16 p., http://www.annales.org/re/2001/re04-2001/gilbert09-24.pdf

GODÉ C. (2012), « Compétences collectives et retour d'expérience "à chaud". Le cas de l'équipe de voltige de l'armée de l'air », Revue française de gestion, 2012/4 (n²23), pp. 167-180.

GUIHOU X., LAGADEC P. \& LAGADEC E. (2006), « Les crises hors cadres et les grands réseaux vitaux, Katrina ", Mission de retour d'expérience - Fait marquants et pistes de réflexion, EDF, $34 \mathrm{p}$.

ESHGHI K. \& LARSON R. (2008), "Disasters: Lessons from the past 105 years", Disaster Prevention \& Management 17, pp. 6282.

KRUKE B. I. \& OLSEN O. E. (2005), "Reliability-seeking networks in complex emergencies", International Journal of Emergency Management 2(4), pp. 275-291.

LALOUETTE C. (2008), " Sécurité industrielle et sous-traitance dans l'aéronautique : comment renforcer le REX ? ", Les Cahiers de la Sécurité industrielle, 5, FONCSI.

LEGENDRE Y. \& GUILLEN L. (2017), « Caractérisation des impacts liés à la houle sur le littoral guadeloupéen au passage de l'ouragan Maria », rapport final, BRGM/RP-67519-FR, 25 illustrations, 10 tableaux, 4 annexes, $209 \mathrm{p}$.

MÉTÉO-FRANCE (2017a), " Passage de l'ouragan exceptionnel Irma sur les îles françaises des Antilles, les 5 et 6 septembre $2017 »$, communiqué de presse.

MÉTÉO-FRANCE (2017b), « Passage de l'ouragan majeur Maria sur les îles françaises des Antilles, les 18 et 19 septembre 2017 ", rapport météorologique.

MIAT (2006), « La conduite du retour d'expérience - Éléments de culture professionnelle ", ministère de l'Intérieur et de l'Aménagement du territoire -Direction de la Défense et de la sécurité civiles

- Sous-direction de la Gestion des risques - Bureau de l'Analyse et de la préparation aux crises, $21 \mathrm{p}$.

MOATTY A. \& VINET F. (2016), Post-disaster recovery: the challenge of anticipation, E3S Web of Conferences7, 17003, FLOODrisk $3^{\text {rd }}$ European Conference on Flood Risk Management, 11 p., doi: 10.1051/e3sconf/20160717003 (https://doi. org/10.1051/e3sconf/20160717003).
MORTUREUX Y. (2004), " Le retour d'expérience en questions », Techniques de l'ingénieur, AG 4608, http//www.techniques-ingénieur.fr/dossier/le_retour_d_expérience_en_questions :SE1040

POURINET L. \& CREACH A. (2019), http://submersions.coselmar.fr/. Les résultats présentés sur ce site sont issus de la thèse de CREACH A. (2015), "Cartographie et analyse économique de la vulnérabilité du littoral atlantique français face au risque de submersion marine ", thèse de doctorat en géographie et économie, Université de Nantes, https://tel.archives-ouvertes. fr/tel-01275600/. Cette thèse a été préparée au sein du laboratoire LETG-Nantes Géolittomer (Unité mixte de Recherche 6554-CNRS-Université de Nantes).

QUIBIER S. (2007), Précurseurs et événements redoutés, recherche et identification, dans le cadre du programme Retour d'expérience par l'analyse systématique des vols, mémoire de DU, Université Paris-Descartes.

VASHDI D., BAMBERGER P., EREZ M. \& WEISS-MEILIK A. (2007), "Briefing-Debriefing: Using are flexive organizational learning model from the military to enhance the performance of surgical teams", Human Resource Management, vol. 46, $\mathrm{n}^{\circ} 1$, pp. 115-142.

WIDALVSKY A. (1991), Searching for safety, New Brunswick, Transaction Publisher.

XIANG Y. \& ZHUANG J. (2014), "A medical resource allocation model for serving emergency victims with deteriorating health conditions", Annals of Operations Research, pp. 1-20.

WYBO J.-L., COLARDELLE C., POULOSSIER M. P. \& CAUCHOIS D. (2001), "Retour d'expérience et gestion des risques. Récents progrès en génie des procédés ", Techniques et Documentation, vol. $15, n^{\circ} 85$, pp. 115-128.

WYBO J.-L. (2004), « Maîtriser les risques de dommages et les risques de crise - Le rôle de l'apprentissage organisationnel ", Risques, $\mathrm{n}^{\circ} 60$, octobre-décembre, pp. 148-157.

WYBO J.-L. \& LATIERS M. (2006), "Exploring complex emergency situations' dynamic: theoretical, epistemological and methodological proposals", International Journal of Emergency Management, vol. $3, n^{\circ} 1$, pp. 40-51. 